\title{
On the mathematical solution of 2D Navier Stokes equations for different geometries
}

\author{
M. A. Mehemed Abughalia \\ Department of Mechanical Engineering, Al-Fateh University, Libya
}

\begin{abstract}
Some analytical solutions of the 1D Navier Stokes equation are introduced in the literature. For 2D flow, the analytical attempts that can solve some of the flow problems sometimes fail to solve more difficult problems or problems of irregular shapes. Many attempts try to simplify the 2D NS equations to ordinary differential equations that are usually solved numerically. The difficulties that are associated with the numerical solution of the Navier Stokes equations are known to the specialists in this field. Some of the problems associated with the numerical solution are; the continuity constraint, pressure-velocity coupling and other problems associated with the mesh generation. This drives the generation of many schemes to simplify and stabilize the 2D Navier Stokes equations. The exact solution of the Navier Stokes equations is difficult and possible only for some cases, mostly when the convective terms vanish in a natural way. This paper is devoted to studying the possibility of finding a mathematical solution of the 2D Navier Stokes equations for both potential and laminar flows. The solutions are a series of functions that satisfy the Navier Stokes equations. The idea behind the solutions is that the complete solution of the $2 \mathrm{D}$ equations is a combination of the solutions of any two terms in the equations; diffusion and advection terms. The solution coefficients should be determined through the boundary conditions.

Keywords: Navier Stokes equations, incompressible flow, fluid flow, Newtonian fluids, potential flow, laminar flow, mathematical solution, steady state.
\end{abstract}

\section{Introduction}

Since the Navier and Stokes derived the mathematical modeling of the fluid in motion; Navier Stokes equations, the mathematical solution have been 
impossible. The difficult in solving these equations prohibited a theoretical treatment of viscous flows. The boundary layer concept, which breakthrough by Prandtl, linked the theory with practice. Prantl showed that the viscous effect is important in a thin region adjacent to a solid.

The governing equations for steady state incompressible flow are the continuity and momentum equations

Continuity equation

$$
\frac{\partial u}{\partial x}+\frac{\partial v}{\partial y}=0
$$

Momentum equations

$$
\begin{gathered}
\rho\left(u \frac{\partial u}{\partial x}+v \frac{\partial u}{\partial y}\right)+\frac{\partial P}{\partial x}=\mu\left(\frac{\partial^{2} u}{\partial x^{2}}+\frac{\partial^{2} u}{\partial y^{2}}\right) \\
\rho\left(u \frac{\partial v}{\partial x}+v \frac{\partial v}{\partial y}\right)+\frac{\partial P}{\partial y}=\mu\left(\frac{\partial^{2} v}{\partial x^{2}}+\frac{\partial^{2} v}{\partial y^{2}}\right)
\end{gathered}
$$

Analytical solution can be obtained for some simple cases and under some assumptions, these equations simplified to get mathematical solution for the boundary layer thickness, shear stress and some basic definitions. To solve the flow over a flat plate, Blasius defined a dimensionless stream function for a laminar flow over flat plate as; [2]

$$
f(\eta)=\frac{\psi}{\sqrt{v x U}}
$$

And by applying the according boundary conditions, the boundary layer thickness is

$$
\delta=\frac{5.0 x}{\sqrt{\mathrm{Re}_{x}}}
$$

And shear stress for laminar flow

$$
\tau_{W}=\frac{0.332 \rho U^{2}}{\sqrt{\operatorname{Re}_{x}}}
$$

For the turbulent flow, mathematical solution shows these functions for the boundary layer thickness and shear stress; [2] 


$$
\delta=\frac{0.382 x}{\operatorname{Re}_{x}^{\frac{1}{5}}}
$$

and shear stress is

$$
\tau_{W}=\frac{0.0594 \rho U^{2}}{\operatorname{Re}_{x}^{\frac{1}{5}}}
$$

Some attempts try to convert these equations into ordinary differential equations. The ordinary differential equations usually solved numerically. Usually, the full solution of the NS equations obtained numerically; Finite difference, finite volume or finite element methods. These numerical methods find difficult in solving the NS equations. The difficulties are due to continuity constraint and strong advection term. Numerically, continuity equation usually replaced by pressure Poisson equation or Penalty function. Many schemes developed to overcome the strong advection term. Some other problems associated with the mesh generation such in dividing the domain into elements or cells with graduate size which not easy. Methods such as; Quad Tree or unstructured grid; are powerful but may fail to discretized domain efficiently. All above is the challenge of this time to find a good scheme or powerful grid generation method.

The present study considers mainly the mathematical solution of twodimensional, steady state laminar flow of Newtonian fluids. The idea that based on is that the full solution of the Navier Stokes equation is a combination of the solution of any two parts. It is known that the solution of the diffusion term is sine and cosine. The advection term solution is exponential.

\section{Mathematical solution of 2D laminar flow equations}

It is possible to find exact solutions for the Navier Stokes equations in certain cases, mostly in which the quadratic convective terms vanishes in a natural way. [5]. Outside the boundary layer, we can suppose that that the viscosity effect is equal in both direction $\mu_{x}=\mu_{y}$ and the diffusion terms are equal in both directions. However, we know the influence of the viscosity confined to the boundary layer.

We can assume a two general function in which one is a solution of the boundary layer and the other is a solution of the free stream potential flow.

We propose that

$$
u=U_{P O}+F(y)+H(x)
$$

Easily we can obtain the following differentiations

$$
\frac{\partial u}{\partial x}=\frac{\partial U_{P O}}{\partial x}+\frac{\partial H(x)}{\partial x}
$$


42 Advances in Fluid Mechanics VI

$$
\begin{aligned}
& \frac{\partial u}{\partial y}=\frac{\partial U_{P O}}{\partial y}+\frac{\partial F(y)}{\partial y} \\
& \frac{\partial^{2} u}{\partial x^{2}}=\frac{\partial^{2} U_{P O}}{\partial x^{2}}+\frac{\partial^{2} H(x)}{\partial x^{2}} \\
& \frac{\partial^{2} u}{\partial y^{2}}=\frac{\partial^{2} U_{P O}}{\partial y^{2}}+\frac{\partial^{2} F(y)}{\partial y^{2}}
\end{aligned}
$$

From the continuity constraint;

$$
\begin{aligned}
& \frac{\partial v}{\partial y}=-\frac{\partial U_{P O}}{\partial x}-\frac{\partial H(x)}{\partial x} \\
& v=V_{P O}+G(x)-\frac{\partial H(x)}{\partial x} y \\
& \frac{\partial v}{\partial x}=\frac{\partial V_{P O}}{\partial x}+G^{\prime}(x)-H^{\prime \prime}(x) y \\
& \frac{\partial^{2} v}{\partial x^{2}}=\frac{\partial^{2} V_{P O}}{\partial x^{2}}+G^{\prime \prime}(x)-H^{\prime \prime \prime}(x) y \\
& \frac{\partial^{2} v}{\partial y^{2}}=\frac{\partial^{2} V_{P O}}{\partial y^{2}}
\end{aligned}
$$

From the first momentum equation, we obtain that;

$$
\begin{gathered}
\frac{\partial P}{\partial x}=-\rho\left(u \frac{\partial u}{\partial x}+v \frac{\partial u}{\partial y}\right)+\mu\left(\frac{\partial^{2} u}{\partial x^{2}}+\frac{\partial^{2} u}{\partial y^{2}}\right) \\
\frac{\partial P}{\partial x}=-\rho\left[U_{P O}+F(y)+H(x)\right] \times\left[\frac{\partial U_{P O}}{\partial x}+H^{\prime}(x)\right] \\
-\rho\left[V_{P O}+G(x)+H^{\prime}(x) y\right] \times\left[\frac{\partial U_{P O}}{\partial y}+F^{\prime}(y)\right] \\
+\mu\left[\frac{\partial^{2} U_{P O}}{\partial x^{2}}+\frac{\partial^{2} U_{P O}}{\partial y^{2}}+F^{\prime \prime}(y)+H^{\prime \prime}(x)\right]
\end{gathered}
$$

By integrate the pressure gradient from, eqn. (20), with respect to $\mathrm{x}$, we obtain that 


$$
\begin{aligned}
x P= & -\rho \int U_{P O} \times \frac{\partial U_{P O}}{\partial x} d x-\rho \int F(y) \times \frac{\partial U_{P O}}{\partial x} d x-\rho \int H(x) \times \frac{\partial U_{P O}}{\partial x} d x \\
& -\rho \int U_{P O} \times H^{\prime}(x) d x-\rho \int F(y) \times H^{\prime}(x) d x-\rho \int H(x) \times H^{\prime}(x) d x \\
& -\rho \int V_{P O} \times \frac{\partial U_{P O}}{\partial y} d x-\rho \int G(x) \times \frac{\partial U_{P O}}{\partial y} d x-\rho \int-H^{\prime}(x) y \times \frac{\partial U_{P O}}{\partial y} d x \\
& -\rho \int V_{P O} \times F^{\prime}(y) d x-\rho \int G(x) \times F^{\prime}(y) d x-\rho \int-H^{\prime}(x) y \times F^{\prime}(y) d x \\
& +\mu\left[F^{\prime \prime}(y) x+H^{\prime}(x)\right]+M(y)
\end{aligned}
$$

Thus

$$
\begin{aligned}
x P= & -\rho U_{P O}{ }^{2}-\rho F(y) \times U_{P O}-\rho H(x) \times U_{P O}+\rho \int U_{P O} \times H^{\prime}(x) d x \\
& -\rho \int U_{P O} \times H^{\prime}(x) d x-\rho F(y) \times H(x)-\rho H(x)^{2} \\
& -\rho \int V_{P O} \times \frac{\partial U_{P O}}{\partial y} d x-\rho \int G(x) \times \frac{\partial U_{P O}}{\partial y} d x+\rho \int H^{\prime}(x) y \times \frac{\partial U_{P O}}{\partial y} d x \\
& -\rho F^{\prime}(y) \times \int V_{P O} d x-\rho F^{\prime}(y) \times \int G(x) d x+\rho y F^{\prime}(y) H(x) \\
& +\mu\left[F^{\prime \prime}(y) x+H^{\prime}(x)\right]+M(y)
\end{aligned}
$$

Rearranging the equation eqn.(22)

$$
\begin{aligned}
x P= & -\rho U_{P O}{ }^{2}-\rho F(y) \times U_{P O}-\rho H(x) \times U_{P O}-\rho F(y) \times H(x) \\
& -\rho H(x)^{2}-\rho V_{P O}{ }^{2}-\rho G(x) \times V_{P O}+\rho \int G^{\prime}(x) \times V_{P O} d x \\
& +\rho \times H^{\prime}(x) y V_{P O}-\rho \int H^{\prime \prime}(x) y \times V_{P O} d x \\
& -\rho F^{\prime}(y) \times \int V_{P O} d x-\rho F^{\prime}(y) \times \int G(x) d x+\rho y F^{\prime}(y) H(x) \\
& +\mu\left[F^{\prime \prime}(y) x+H^{\prime}(x)\right]+M(y)
\end{aligned}
$$

By applying the expressions for the velocities and their derivatives into the second momentum equation eqn.(3).

$$
\begin{gathered}
\frac{\partial P}{\partial y}=-\rho\left(u \frac{\partial v}{\partial x}+v \frac{\partial v}{\partial y}\right)+\mu\left(\frac{\partial^{2} v}{\partial x^{2}}+\frac{\partial^{2} v}{\partial y^{2}}\right) \\
\frac{\partial P}{\partial y}=-\rho\left[U_{P O}+F(y)+H(x)\right] \times\left[\frac{\partial V_{P O}}{\partial x}+G^{\prime}(x)-H^{\prime \prime}(x) y\right] \\
-\rho\left[V_{P O}+G(x)-\frac{\partial H(x)}{\partial x} y\right] \times\left[-\frac{\partial U_{P O}}{\partial x}-\frac{\partial H(x)}{\partial x}\right] \\
+\mu\left[\frac{\partial^{2} V_{P O}}{\partial x^{2}}+G^{\prime \prime}(x)-H^{\prime \prime \prime}(x) y+\frac{\partial^{2} V_{P O}}{\partial y^{2}}\right]
\end{gathered}
$$


By integrate the pressure gradient from, eqn.(25), with respect to y, we obtain that

$$
\begin{aligned}
y P & =-\rho \int U_{P O} \times \frac{\partial V_{P O}}{\partial x} d y-\rho \int F(y) \times \frac{\partial V_{P O}}{\partial x} d y-\rho \int H(x) \times \frac{\partial V_{P O}}{\partial x} d y \\
& -\rho \int U_{P O} \times G^{\prime}(x) d y-\rho \int F(y) \times G^{\prime}(x) d y \\
& -\rho \int H(x) \times G^{\prime}(x) d y \\
& -\rho \int U_{P O} \times-H^{\prime \prime}(x) y d y-\rho \int F(y) \times-H^{\prime \prime}(x) y d y \\
& -\rho \int H(x) \times-H^{\prime \prime}(x) y d y \\
& +\rho \int V_{P O} \times \frac{\partial U_{P O}}{\partial x} d y+\rho \int G(x) \times \frac{\partial U_{P O}}{\partial x} d y \\
& +\rho \int-\frac{\partial H(x)}{\partial x} y \times \frac{\partial U_{P O}}{\partial x} d y \\
& +\rho \int V_{P O} \times \frac{\partial H(x)}{\partial x} d y+\rho \int G(x) \times \frac{\partial H(x)}{\partial x} d y \\
& +\rho \int-\frac{\partial H(x)}{\partial x} y \times \frac{\partial H(x)}{\partial x} d y \\
& +\mu \int\left[\frac{\partial^{2} V_{P O}}{\partial x^{2}}+G^{\prime \prime}(x)-H^{\prime \prime \prime}(x) y+\frac{\partial^{2} V_{P O}}{\partial y^{2}}\right] d y
\end{aligned}
$$

which can be re-expressed as:

$$
\begin{aligned}
y P= & -\rho U_{P O}{ }^{2}-\rho F(y) \times U_{P O}+\rho \int F^{\prime}(y) \times U_{P O} d y-\rho H(x) \times U_{P O} \\
& -\rho G^{\prime}(x) \times \int U_{P O} d y-\rho G^{\prime}(x) \times \int F(y) d y-\rho G^{\prime}(x) \times H(x) y \\
& +\rho H^{\prime \prime}(x) \int U_{P O} \times y d y+\rho H^{\prime \prime}(x) \int F(y) \times y d y \\
& +\rho H^{\prime \prime}(x) H(x) \frac{y^{2}}{2} \\
& -\rho V_{P O}{ }^{2}-\rho G(x) \times V_{P O}+\rho \times H^{\prime}(x) y V_{P O} \\
& +\rho G(x) \times H^{\prime}(x) y+\rho\left[H^{\prime}(x)\right]^{2} \frac{y^{2}}{2} \\
& +\mu\left[G^{\prime \prime}(x) y-H^{\prime \prime \prime}(x) \frac{y^{2}}{2}\right]+N(x)
\end{aligned}
$$

To make the diffusion terms equal in eqn.(23) \& eqn.(27); we get;

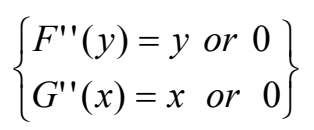


Therefore, for the advection terms, we get the following conditions:

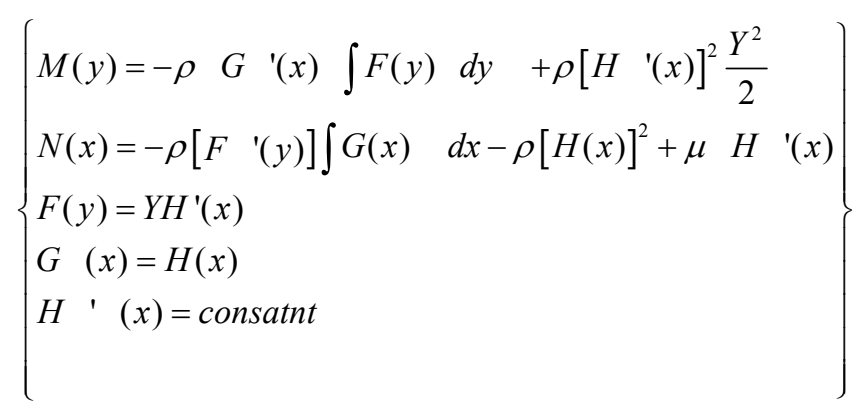

The solution for the irrotational laminar flow is:

$$
\begin{aligned}
& u=U_{P O}+H^{\prime}(x) y+H(x) \\
& v=V_{P O}+H(x)-\frac{\partial H(x)}{\partial x} y
\end{aligned}
$$

The solution for the rotational laminar flow is:

$$
\begin{gathered}
u=U_{P O}+F(y) \\
v=V_{P O}+G(x)
\end{gathered}
$$

where

$$
\begin{gathered}
H(x)=0, F^{\prime}(y)=y \text { and } G^{\prime}(x)=x \\
U_{P O}=? \\
V_{P O}=?
\end{gathered}
$$

The pressure distribution for both rotational and irrotational flows is:

$$
\begin{aligned}
P= & -\rho\left[U_{P O}\right]^{2}-\rho \times F(y) U_{P O}+\rho \int F^{\prime}(y) \times U_{P O} d y \\
& -\rho H(x) \times U_{P O}-\rho G^{\prime}(x) \int U_{P O} d y \\
& -\rho G^{\prime}(x) \int F(y) d y-\rho G^{\prime}(x) H(x) Y-\rho\left[V_{P O}\right]^{2} \\
& -\rho G(x) \times V_{P O}+\rho H^{\prime}(x) Y \times V_{P O} \\
& +\rho H^{\prime}(x) G(x) Y+\rho\left[H^{\prime}(x)\right]^{2} \frac{Y^{2}}{2} \\
& +N(x)+\mu\left(G^{\prime \prime}(x) Y\right)
\end{aligned}
$$


The rotation function is defined by [2]

$$
\begin{gathered}
\omega=\frac{1}{2}\left(\frac{\partial \mathrm{v}}{\partial \mathrm{x}}-\frac{\partial \mathrm{u}}{\partial \mathrm{y}}\right) \\
\omega=\frac{1}{2}\left[\left[\frac{\partial V_{P O}}{\partial x}+G^{\prime}(x)-H^{\prime \prime \prime}(x) y\right]-\left[\frac{\partial U_{P O}}{\partial y}+F^{\prime}(y)\right]\right]
\end{gathered}
$$

The shear stress; [2]

$$
\begin{gathered}
\tau_{x y}=\mu\left(\frac{\partial \mathrm{v}}{\partial \mathrm{x}}+\frac{\partial \mathrm{u}}{\partial \mathrm{y}}\right) \\
\tau_{x y}=\mu\left[\frac{\partial V_{P O}}{\partial x}+\frac{\partial U_{P O}}{\partial y}+G^{\prime}(x)+F^{\prime}(y)\right]
\end{gathered}
$$

At the walls, the velocity components are equal to zero, so we have that

$$
\begin{aligned}
& \frac{\partial P}{\partial x}=-\rho\left(u \frac{\partial u}{\partial x}+v \frac{\partial u}{\partial y}\right)+\mu\left(\frac{\partial^{2} u}{\partial x^{2}}+\frac{\partial^{2} u}{\partial y^{2}}\right)=0 \\
& \frac{\partial P}{\partial y}=-\rho\left(u \frac{\partial v}{\partial x}+v \frac{\partial v}{\partial y}\right)+\mu\left(\frac{\partial^{2} v}{\partial x^{2}}+\frac{\partial^{2} v}{\partial y^{2}}\right)=0
\end{aligned}
$$

\section{Conclusion and future work}

The mathematical background of the problem will help in the numerical study. In finite element, the element type should be chosen according to the physical problem. The mathematical solution that considered here is a continuous function over the domain; inside and outside the boundary layer; and satisfies continuity and momentum equations. The coefficients included in the solution should be determined through satisfying the boundary conditions for velocity and pressure. But the boundary condition for the pressure at the wall is satisfied already as the boundary condition for the velocity applied. Therefore, the boundary conditions for the velocity just needed to be satisfied.

This solution could be a general solution for potential and laminar flows. The shear between the fluid layers does not vanish outside the boundary layer because the friction between the layers cannot be eliminated. The advantage of the mathematical solution over the numerical solutions is so clear, but can be obtained for simple geometries. A future work will consider the application of the solution for simple cases and a solution for the thermal and turbulent flows will be brought. 


\section{References}

[1] W. M. Kays, M. E. Crawford, Convective Heat and Mass Transfer, McGraw Hill, New York, 1993.

[2] Robert, W. Fox, Alan T. McDonald, Introduction to Fluid Mechanics, John Wiley \& Sons, 1994.

[3] H. Bateman, Partial Differential Equations of Mathematical Physics, Cambridge University press 1964.

[4] A.J. Baker, Finite element Computational Fluid Mechanics, Hemisphere, 1983.

[5] Hermann Schlichting, Boundary Layer Theory, McGraw-Hill, 1968. 